\title{
NON-UNIQUENESS AND PRESCRIBED ENERGY FOR THE CONTINUITY EQUATION
}

\author{
GIANLUCA CRIPPA, NIKOLAY GUSEV, STEFANO SPIRITO, AND EMIL WIEDEMANN
}

ABStract. In this note we provide new non-uniqueness examples for the continuity equation by constructing infinitely many weak solutions with prescribed energy.

\section{INTRODUCTION}

In this paper we consider the continuity equation for a bounded scalar function $u: \mathbb{R} \times \mathbb{R}^{d} \rightarrow \mathbb{R}$ with a bounded divergence-free vector field $\mathbf{b}: \mathbb{R} \times \mathbb{R}^{d} \rightarrow \mathbb{R}^{d}$ :

$$
\begin{gathered}
\partial_{t} u+\operatorname{div}(u \mathbf{b})=0, \\
\operatorname{div} \mathbf{b}=0 .
\end{gathered}
$$

This equation appears in various problems of mathematical physics, in particular fluid mechanics and kinetic theory. In the smooth setting (and assuming suitable integrability) the energy

$$
\mathscr{E}(t):=\int_{\mathbb{R}^{d}} u^{2}(t, x) d x
$$

of the solution $u$ is conserved:

$$
\frac{d}{d t} \mathscr{E}(t)=0
$$

Indeed, since $\mathbf{b}$ is divergence-free, by multiplying (1) with $u$, using the chain rule and integrating over $\mathbb{R}^{d}$ one immediately obtains $(3)$.

In many applications one has to study (1) in a nonsmooth setting. Roughly speaking, since (1) is linear, the conservation of energy (3) implies uniqueness of weak solutions to the corresponding initial-value problem for (1). In fact, conservation of energy is a consequence of the so-called renormalization property, which was proved by [DL89] for any vector field b with Sobolev regularity and later extended by Ambrosio $\mathrm{Amb}$ to the case when $b$ has bounded variation. We refer to DL08, AC14 for a detailed review of recent results in this direction.

On the other hand, when the regularity of the vector field $\mathbf{b}$ is too low, the conservation of energy (3) fails in general. In a nonsmooth setting several counterexamples to the uniqueness, and therefore to the conservation of energy, are known, see [Aiz78, CLR03, Dep03, ABC14, ABC13. A similar phenomenon occurs in the context of the Euler equations. For example, in the papers [Sch93, Shn97, DLS09] weak solutions of the Euler equations were constructed with compact support in space time.

In particular the example in Dep03 gives a bounded vector field $\mathbf{b}$ and a bounded scalar field $u$ which satisfy (1) and (2) such that

$$
\mathscr{E}(t)= \begin{cases}0 & \text { for } t \leq 0 \\ 1 & \text { for } t>0\end{cases}
$$

2010 Mathematics Subject Classification. Primary: 35F10, Secondary: 35A02.

Key words and phrases. Transport and continuity equations, Non-uniqueness, Non-conservation of energy, Renormalization, Convex integration. 
In this paper, for any given nonnegative bounded function $E: \mathbb{R} \rightarrow \mathbb{R}$ which is continuous on an open interval and zero outside we construct infinitely many pairs (b, $u$ ) satisfying (1) and (2), such that $\mathscr{E}(t)=E(t)$ for a.e. $t$. Thus, in contrast with (4), we provide more general profiles for the energy. Our results are also connected to the chain rule problem for the divergence operator, see ADLM07, BG14, CGSW].

We construct such pairs $(\mathbf{b}, u)$ using the method of convex integration, and our techniques are similar to the ones used in DLS09, Szé12. The latter reference contains an appendix giving a general framework for convex integration, but for the problem at hand we need to consider a nonlinear constraint that depends on the points in the domain (as was the case e.g. in [DLS10], albeit in a different functional setting). For this reason we adapt the framework from Szé12 to this more general situation (see $\$ 2$ ). We then apply this abstract framework to the specific situation of the continuity equation (see $\$ 3$ ).

Finally, let us mention [CFG11, Shv11, BLFNL, where results were obtained by convex integration, respectively, that yield as a byproduct counterexamples to the energy conservation for continuity equations. However, in these works the energy profile is always piecewise constant.

Acknowledgements. This research has been partially supported by the SNSF grants 140232 and 156112. This work was started while the third author was a PostDoc at the Departement Mathematik und Informatik of the Universität Basel. He would like to thank the department for the hospitality and the support. The second author was partially supported by the Russian Foundation for Basic Research, project no. 13-01-12460. The authors are grateful to S. Bianchini, C. De Lellis, and L. Székelyhidi for the fruitful discussions about the topic of the paper.

\section{Differential INCLUSIONS With NON-CONSTANT NONLINEAR CONSTRAinT}

We start with the so-called Tartar framework (cf. e.g. [DLS09]). Consider a system of $m$ linear partial differential equations

$$
\sum_{i=1}^{D} A_{i} \partial_{i} z=0
$$

in an open set $\mathscr{D} \subset \mathbb{R}^{D}$, where $A_{i}$ are constant $m \times n$ matrices and $z: \mathscr{D} \rightarrow \mathbb{R}^{n}$. Consider a nonlinear constraint

$$
z(y) \in K_{y}
$$

for a.e. $y$ in $\mathscr{D}$, where $K_{y} \subset \mathbb{R}^{n}$ is a compact set for any $y \in \mathscr{D}$.

For any $y \in \mathscr{D}$ let $U_{y}:=\operatorname{int} \operatorname{conv} K_{y}$, where with conv we denote the convex hull of the set $K_{y}$ and with int we denote its interior. Let $\mathscr{U} \subset \mathscr{D}$ be a bounded open set.

Definition 1 (Subsolutions). We say that $z \in L^{2}(\mathscr{D})$ is a subsolution of (5), (6) if $z$ is a weak solution of (5) in $\mathscr{D}, z$ is continuous on $\mathscr{U}$, (6) holds for a.e. $y \in \mathscr{D} \backslash \mathscr{U}$ and

$$
z(y) \in U_{y}
$$

for any $y \in \mathscr{U}$.

Definition 2 (Localized plane waves/wave cone). A set $\Lambda \subset \mathbb{R}^{n}$ is called wave cone if there exists a constant $C>0$ such that for any $\bar{z} \in \Lambda$ there exists a sequence $w_{k} \in C_{0}^{\infty}\left(B_{1}(0) ; \mathbb{R}^{n}\right)$ solving (5) in $\mathbb{R}^{D}$ such that

- $\operatorname{dist}\left(w_{k}(x),[-\bar{z}, \bar{z}]\right) \rightarrow 0$ for all $x \in B_{1}(0)$ uniformly as $k \rightarrow \infty$,

- $w_{k} \rightarrow 0$ in $L^{2}$ as $k \rightarrow \infty$,

- $\int\left|w_{k}\right|^{2} d y>C|\bar{z}|^{2}$ for all $k \in \mathbb{N}$. 
In the above definition we denoted the segment with endpoints $x \in \mathbb{R}^{n}$ and $y \in \mathbb{R}^{n}$ with $[x, y]:=\operatorname{conv}\{x, y\}$. The functions $w_{k}$ are called localized plane waves. We make the following assumptions:

Assumption 1. There exists a wave cone $\Lambda$ dense in $\mathbb{R}^{n}$.

Let $\mathscr{K}$ denote the set of all compact subsets of $\mathbb{R}^{n}$, endowed with the Hausdorff metric $d_{\mathcal{H}}$. It is well-known that $\mathscr{K}$ is a complete metric space.

Assumption 2 (Continuity of the nonlinear constraint). The map $f: \mathscr{U} \ni y \mapsto K_{y} \in \mathscr{K}$ is continuous and bounded in the Hausdorff metric.

Our main abstract result is the following:

Theorem 1. Suppose that Assumptions 1 and 2 hold. Suppose that $z_{0}$ is a subsolution of (5), (6). Then there exist infinitely many weak solutions $z \in L^{2}(\mathscr{D})$ of (5) which agree with $z_{0}$ a.e. on $\mathscr{D} \backslash \mathscr{U}$ and satisfy (6) for a.e. $y \in \mathscr{D}$.

2.1. Geometric preliminaries. The next lemma shows that compact subsets of the interior of the convex hull of a compact set $K$ are stable with respect to sufficiently small perturbations of $K$ in the Hausdorff metric.

Lemma 1. Let $K \subset \mathbb{R}^{n}$ be a compact set. Then for any compact set $C \subset \operatorname{int} \operatorname{conv} K$ there exists $\varepsilon>0$ such that for any compact set $K^{\prime} \subset \mathbb{R}^{n}$ with $d_{\mathcal{H}}\left(K, K^{\prime}\right)<\varepsilon$ we have

$$
C \subset \operatorname{int} \operatorname{conv} K^{\prime} .
$$

Figure 1. An illustration of Lemma 1 in the case when $K=\{L, M, N\}$ and $K^{\prime}=\left\{L^{\prime}, M^{\prime}, N^{\prime}\right\}$.



Proof. Since int conv $K$ is open, for any point $x \in C$ there exists a simplex $S_{x}$ with vertices $\left\{v_{i}\right\}_{i=1 . . n+1} \subset$ conv $K$ such that $x$ belongs to the inner open simplex

$$
I_{x}:=\left\{\sum_{i=1}^{n+1} \lambda_{i} v_{i} \mid \lambda_{i} \in\left(\frac{1}{2(n+1)}, \frac{2}{n+1}\right), \sum_{i=1}^{n+1} \lambda_{i}=1, i=1 . . n+1\right\} .
$$

Since $C$ is a compact set and the inner simplices $\left\{I_{x}\right\}_{x \in C}$ cover $C$ we can extract a finite subcover $\left\{I_{x_{k}}\right\}_{k=1 . . m}$ of $C$. Let us fix $k \in 1 . . m$ and consider the simplex $S:=S_{x_{k}}$ with vertices $\left\{v_{i}\right\}_{i=1 . . n+1} \subset$ conv $K$. Let $I:=I_{x_{k}}$ denote the corresponding inner simplex. 
If $\varepsilon<\operatorname{dist}(\partial I, \partial S)$ then for any points $v_{i}^{\prime} \in B_{\varepsilon}\left(v_{i}\right), i=1 . . n+1$ one has

$$
I \subset \operatorname{conv}\left\{v_{1}^{\prime}, v_{2}^{\prime}, \ldots, v_{n+1}^{\prime}\right\} .
$$

Observe that for any $\varepsilon>0$ and $i=1 . . n+1$ the ball $B_{\varepsilon}\left(v_{i}\right)$ contains a point $v_{i}^{\prime} \in \operatorname{conv} K^{\prime}$. Indeed, by Caratheodory's theorem $v_{i}=\sum_{j=1}^{n+1} \mu_{j} z_{j}$ for some $z_{j} \in K$ and $\mu_{j} \in[0,1]$ with $\sum_{j=1}^{n+1} \mu_{j}=1$. Since $d_{\mathcal{H}}\left(K, K^{\prime}\right)<\varepsilon$ there exist points $z_{j}^{\prime} \in K^{\prime}$ such that $z_{j}^{\prime} \in B_{\varepsilon}\left(z_{j}\right)$, where $j=1 . . n+1$. Let

$$
v_{i}^{\prime}:=\sum_{j=1}^{n+1} \mu_{j} z_{j}^{\prime},
$$

then $\left|v_{i}-v_{i}^{\prime}\right| \leq \sum_{j=1}^{n+1} \mu_{j}\left|z_{j}-z_{j}^{\prime}\right|<\varepsilon$. Hence by (8) we have $I \subset \operatorname{conv}\left\{v_{1}^{\prime}, v_{2}^{\prime}, \ldots, v_{n+1}^{\prime}\right\}$ provided that $\varepsilon$ is small enough. But $v_{i}^{\prime} \in \operatorname{conv} K^{\prime}$, hence $I \subset \operatorname{conv} K^{\prime}$. Since $I$ is open we can also write $I \subset \operatorname{int} \operatorname{conv} K^{\prime}$.

Since we have finitely many simplices, we can choose $\varepsilon>0$ in such a way that the inclusion $I_{x_{k}} \subset \operatorname{int}$ conv $K^{\prime}$ holds for any $k=1 . . m$ (provided that $d_{\mathcal{H}}\left(K, K^{\prime}\right)<\varepsilon$ ). Then

$$
C \subset \cup_{k=1 . . m} I_{x_{k}} \subset \operatorname{int} \operatorname{conv} K^{\prime} .
$$

We will also need the following elementary lemma:

Lemma 2. Suppose that $z \in C\left(\mathscr{U} ; \mathbb{R}^{n}\right)$ where $\mathscr{U} \subset \mathbb{R}^{D}$ is an open set. Suppose that for any $y \in \mathscr{U}$ we have a compact set $K_{y} \subset \mathbb{R}^{n}$ and the function $y \mapsto K_{y}$ is continuous in the Hausdorff metric. Then the function $F: y \mapsto \operatorname{dist}\left(z(y), K_{y}\right)$ is continuous on $\mathscr{U}$.

Proof. One can prove directly that the function $(z, K) \mapsto \operatorname{dist}(z, K)$ is continuous on $\mathbb{R}^{n} \times \mathscr{K}$. The function $y \mapsto\left(z(y), K_{y}\right)$ is continuous in view of the assumptions. Hence the function $F$ is continuous as a composition of continuous functions.

In general the distance from a point $z$ to a compact set $K$ does not control from below the distance from $z$ to the boundary of conv $K$. However the following lemma shows that there exists a segment inside int conv $K$ with midpoint $z$ and length controlled from below by $\operatorname{dist}(z, K)$ :

Lemma 3 (Geometric lemma). Let $K \subset \mathbb{R}^{n}$ be a compact set. For any $z \in \operatorname{int}$ conv $K$ there exists $\bar{z} \in \mathbb{R}^{n}$ such that

- $[z-\bar{z}, z+\bar{z}] \subset \operatorname{int} \operatorname{conv} K$

- $|\bar{z}| \geq \frac{1}{2 n} \operatorname{dist}(z, K)$

(This is exactly Lemma 5.3 from DLS12.)

2.2. Convex integration. The following lemma is the main building block of the convex integration scheme:

Lemma 4 (Perturbation lemma). Suppose that Assumptions 1 and 2 hold and $z$ is a subsolution of (5) and (6) such that

$$
\int_{\mathscr{U}} \operatorname{dist}^{2}\left(z(y), K_{y}\right) d y=\varepsilon>0 .
$$

Then there exists $\delta=\delta(\varepsilon)>0$ and a sequence $\left\{z_{k}\right\}_{k \in \mathbb{N}}$ of subsolutions of (5) and (6) such that

- $z_{k}=z$ on $\mathscr{D} \backslash \mathscr{U}$ for any $k \in \mathbb{N}$

- $\int_{\mathscr{U}}\left|z-z_{k}\right|^{2} d y \geq \delta$ for any $k \in \mathbb{N}$

- $z_{k} \rightarrow z$ in $L^{2}(\mathscr{U})$ as $k \rightarrow \infty$. 
Proof. Step 1. Let $y \in \mathscr{U}$. Since $z(y) \in U_{y}$ we can apply Lemma 3 to obtain $\bar{z}_{*}(y)$ such that

$$
\begin{gathered}
{\left[z(y)-\bar{z}_{*}(y), z(y)+\bar{z}_{*}(y)\right] \subset U_{y},} \\
\left|\bar{z}_{*}(y)\right| \geq \frac{1}{2 n} \operatorname{dist}\left(z(y), K_{y}\right),
\end{gathered}
$$

Since $\Lambda$ is dense in $\mathbb{R}^{n}$ and $U_{y}$ is open we can find $\bar{z}(y) \in \Lambda$ such that

$$
\begin{gathered}
{[z(y)-\bar{z}(y), z(y)+\bar{z}(y)] \subset U_{y},} \\
|\bar{z}(y)| \geq \frac{1}{4 n} \operatorname{dist}\left(z(y), K_{y}\right) .
\end{gathered}
$$

Due to $(9)$ there exists $\rho(y)>0$

$$
[z(y)-\bar{z}(y), z(y)+\bar{z}(y)]+\overline{B_{2 \rho(y)}(0)} \subset U_{y} .
$$

Hence using Assumption 2, Lemma 1 and the continuity of $z$ we can find $R(y)>0$ such that

$$
[z(x)-\bar{z}(y), z(x)+\bar{z}(y)]+\overline{B_{\rho(y)}(0)} \subset U_{x}
$$

for all $x \in B_{R(y)}(y) \subset \mathscr{U}$. Moreover, in view of Lemma 2 we can choose $R(y)$ in such a way that

$$
\operatorname{dist}\left(z(x), K_{x}\right) \leq 2 \operatorname{dist}\left(z(y), K_{y}\right)
$$

for all $x \in B_{R(y)}(y)$.

Using Assumption 1 for any fixed $y \in \mathscr{U}$ we can construct a sequence $\left\{w_{y, k}\right\}_{k \in \mathbb{N}} \subset C_{0}^{\infty}\left(B_{1}(0)\right)$ such that

- $w_{y, k}(x) \in[-\bar{z}(y), \bar{z}(y)]+B_{\rho(y)}(0)$ for all $x \in B_{1}(0)$ and $k \in \mathbb{N}$,

- $w_{y, k} \rightarrow 0$ in $L^{2}$ as $k \rightarrow \infty$,

- $\int\left|w_{y, k}\right|^{2} d x>C|\bar{z}(y)|^{2}$ for all $k \in \mathbb{N}$.

Step 2. Let $\varepsilon:=\int_{\mathscr{U}} \operatorname{dist}^{2}\left(z(y), K_{y}\right) d y$. The balls $\left\{B_{r}(y) \mid y \in \mathscr{U}, r \in(0, R(y))\right\}$ cover $\mathscr{U}$, so using Vitali's covering theorem (see e.g. Bog07], Theorem 5.5.2) and the absolute continuity of the Lebesgue integral we can find finitely many points $\left\{y_{i}\right\}_{i=1 . . N} \subset \mathscr{U}$ and radii $r_{i} \in\left(0, R\left(y_{i}\right)\right)$ such that

$$
\sum_{i=1}^{N} \int_{B_{i}} \operatorname{dist}^{2}\left(z(y), K_{y}\right) d y>\frac{1}{2} \varepsilon
$$

where the balls $B_{i}:=B_{r_{i}}\left(y_{i}\right)$ are pairwise disjoint.

For each $i=1 . . N$ let us introduce the scaled and translated perturbations $w_{i, k}(x):=$ $w_{y_{i}, k}\left(\frac{x-y_{i}}{r_{i}}\right)$. These functions belong to $C_{0}^{\infty}\left(B_{i}\right)$ and satisfy

(i) $w_{i, k}(x) \in\left[-\bar{z}\left(y_{i}\right), \bar{z}\left(y_{i}\right)\right]+B_{\rho\left(y_{i}\right)}(0)$ for all $x \in B_{i}, k \in \mathbb{N}, i=1 . . N$;

(ii) $w_{i, k} \rightarrow 0$ in $L^{2}$ as $k \rightarrow \infty$ (for each fixed $i=1 . . N$ );

(iii) $\int\left|w_{i, k}\right|^{2} d x>C\left|\bar{z}\left(y_{i}\right)\right|^{2} \mathscr{L}^{D}\left(B_{i}\right)$ for all $k \in \mathbb{N}$.

In view of (i) and (11) we have $z(x)+w_{i, k}(x) \in U_{x}$ for all $x \in \mathscr{U}$ and $i=1 . . N$, hence $z+w_{i, k} \in X_{0}$. Since the balls $B_{i}$ are pairwise disjoint the function

$$
z_{k}:=z+\sum_{i=1}^{N} w_{i, k}
$$

also belongs to $X_{0}$. 
Using successively (iii), (10), (12) and (13) we obtain:

$$
\begin{aligned}
& \int_{\mathscr{U}}\left|z-z_{k}\right|^{2} d y=\sum_{i=1}^{N} \int_{B_{i}}\left|w_{i, k}(y)\right|^{2} d y \stackrel{(\mathrm{iii})}{>} C \sum_{i=1}^{N}\left|\bar{z}\left(y_{i}\right)\right|^{2} \mathscr{L}^{D}\left(B_{i}\right) \\
& \stackrel{100}{\geq} \frac{C}{16 n^{2}} \sum_{i=1}^{N} \operatorname{dist}^{2}\left(z\left(y_{i}\right), K_{y_{i}}\right) \mathscr{L}^{D}\left(B_{i}\right)=\frac{C}{16 n^{2}} \sum_{i=1}^{N} \int_{B_{i}} \operatorname{dist}^{2}\left(z\left(y_{i}\right), K_{y_{i}}\right) d x \\
& \stackrel{120}{>} \frac{C}{32 n^{2}} \sum_{i=1}^{N} \int_{B_{i}} \operatorname{dist}^{2}\left(z(x), K_{x}\right) d x \stackrel{\text { 13) }}{>} \frac{C}{64 n^{2}} \varepsilon
\end{aligned}
$$

It remains to observe that since $N$ is finite and the points $y_{i}$ are fixed we have $z_{k} \rightarrow z$ in $L^{2}$ as $k \rightarrow \infty$.

\subsection{Proof of Theorem 1. We are now ready to prove our main abstract theorem.}

Proof of Theorem 1. Let $X_{0}$ denote a set of all subsolutions of (5) and (6) which agree with $z_{0}$ on $\mathscr{D} \backslash \mathscr{U}$. Let $X$ be the closure of $X_{0}$ in the weak topology of $L^{2}(\mathscr{U})$, endowed with the corresponding induced weak topology. Clearly any $z \in X$ solves (5) and satisfies (2) a.e. on $\mathscr{D} \backslash \mathscr{U}$.

For any $z \in X$ let us define

$$
I(z):=\int_{\mathscr{U}}|z(y)|^{2} d y
$$

This functional is a Baire-1 function on $X$. Indeed, for any $j \in \mathbb{N}$ let

$$
I_{j}(z):=\int_{\{y \in \mathscr{U} \mid \operatorname{dist}(y, \partial U)>1 / j\}}\left|\left(\omega_{1 / j} * z\right)(y)\right|^{2} d y
$$

where for any $\varepsilon>0$ we denote by $\omega_{\varepsilon}(\cdot)=\varepsilon^{-D} \omega(\cdot / \varepsilon)$ the standard convolution kernel. Then for any $j \in \mathbb{N}$ the functional $I_{j}$ is continuous on $X$, and for any $z \in X$ we have $I_{j}(z) \rightarrow I(z)$ as $j \rightarrow \infty$.

In view of Assumption $2 X$ is a bounded subset of $L^{2}(\mathscr{U})$. Since the weak topology is metrizable on the norm-bounded subsets of $L^{2}(\mathscr{U})$, we can consider $X$ as a complete metric space with some metric $d_{X}$.

Then by Baire category theorem (see also Theorem 7.3 from [Oxt80]) the set

$$
Y:=\{z \in X \mid I \text { is continuous at } z\}
$$

is residual in $X$ (and hence is infinite). We claim that $z \in Y$ implies $J(z)=0$, where

$$
J(z):=\int_{\mathscr{U}} \operatorname{dist}^{2}\left(z(y), K_{y}\right) d y .
$$

Indeed, suppose that $J(z)=\varepsilon>0$ for some $z \in Y$. Let $z_{j} \in X_{0}$ be a sequence such that $z_{j} \rightarrow z$ in $L^{2}(\mathscr{U})$ as $j \rightarrow \infty$. Since $I$ is continuous at $z$ this implies that $I\left(z_{j}\right) \rightarrow I(z)$ and consequently $z_{j} \rightarrow z$ in $L^{2}(\mathscr{U})$ as $j \rightarrow \infty$.

Then in view of Assumption 2 we have $J\left(z_{j}\right) \rightarrow J(z)$ as $j \rightarrow \infty$ and hence without loss of generality we can assume that $J\left(z_{j}\right)>\varepsilon / 2$ for all $j \in \mathbb{N}$.

Applying Lemma 4 to $z_{j}$ for each $j \in \mathbb{N}$ we can find $\widetilde{z}_{j} \in X_{0}$ such that $d_{X}\left(\widetilde{z}_{j}, z_{j}\right)<2^{-j}$ and $\int_{\mathscr{U}}\left|\widetilde{z}_{j}-z_{j}\right|^{2} d y \geq \delta>0$, where $\delta=\delta(\varepsilon)$ is independent of $j$.

Since $d_{X}\left(\widetilde{z}_{j}, z\right) \leq d_{X}\left(\widetilde{z}_{j}, z_{j}\right)+d_{X}\left(z_{j}, z\right) \rightarrow 0$ as $j \rightarrow \infty$ we also have $\widetilde{z}_{j} \rightarrow z$ in $L^{2}$. Since $z$ is a point of continuity of $I$ we also have $z_{j} \rightarrow z$ in $L^{2}(\mathscr{U})$ as $j \rightarrow \infty$. But then $\widetilde{z}_{j}-z_{j} \rightarrow 0$ in $L^{2}(\mathscr{U})$, which contradicts the construction of $\widetilde{z}_{j}$. 


\section{Application to the CONTINUity eQuation}

In this section we apply our abstract framework to the case of the continuity equation.

Theorem 2. Suppose that $d \geq 2$. Let $E: \mathbb{R} \rightarrow \mathbb{R}$ be a non-negative bounded function which is continuous on some bounded open interval $I \subset \mathbb{R}$ and vanishes on $\mathbb{R} \backslash I$. Then there exist infinitely many bounded, compactly supported $u: \mathbb{R} \times \mathbb{R}^{d} \rightarrow \mathbb{R}$ and $\mathbf{b}: \mathbb{R} \times \mathbb{R}^{d} \rightarrow \mathbb{R}^{d}$ which satisfy (1) and (2) in sense of distributions and such that

$$
\int_{\mathbb{R}^{2}} u^{2}(t, x) d x=E(t) \quad \text { for a.e. } t \in I .
$$

Remark 1. It is well-known that a representative of $u$ can be chosen such that the map $t \mapsto u(t, \cdot)$ is continuous with values in $L^{2}$ equipped with the weak topology. Then the question arises whether the assertion in the theorem holds even for every, and not just almost every, time $t$. We expect this to be true: indeed this should follow by methods similar to those of [DLS10]. We will however not pursue this question further in this article.

Remark 2. When $d=2$ and $f$ is a characteristic function of an interval, the statement of Theorem 2, essentially, follows from the example constructed in Dep03. This particular case of Theorem 2 was also proved in Gus11 using the convex integration method.

Remark 3. A similar problem can be addressed for more general equation of the form $\operatorname{div}(u \mathbf{B})=0$ instead of (1). For this equation the problem is stated as follows: given a distribution $g$ is it possible to construct compactly supported bounded functions $u: \mathbb{R}^{n} \rightarrow \mathbb{R}, \mathbf{B}: \mathbb{R}^{n} \rightarrow \mathbb{R}^{n}$ such that

$$
\operatorname{div}(u \mathbf{B})=0, \quad \operatorname{div} \mathbf{B}=0, \quad \operatorname{div}\left(u^{2} \mathbf{B}\right)=g ?
$$

This is related to the so-called chain rule problem for the divergence operator [ADLM07]. When $n=2$ such a construction is not possible for $g \neq 0$ in view of [BG14], while for $n \geq 3$ it is possible and is obtained using convex integration and rank-2 laminates in [CGSW].

Let us put the continuity equation in the framework of the previous section. Fix a bounded open set $\Omega \subset \mathbb{R}^{d}$. Let $\mathscr{U}:=I \times \Omega$ and

$$
F(t, x):=\frac{E(t)}{\mathscr{L}^{d}(\Omega)} \mathbf{1}_{\Omega}(x),
$$

where $\mathbf{1}_{\Omega}$ denotes the characteristic function of $\Omega$.

We consider equations (1) and (2) as a linear system

$$
\begin{gathered}
\partial_{t} u+\operatorname{div}_{x} \mathbf{m}=0, \\
\operatorname{div}_{x} \mathbf{b}=0
\end{gathered}
$$

in $\mathscr{D}:=\mathbb{R} \times \mathbb{R}^{d}$ with $u: \mathscr{D} \rightarrow \mathbb{R}, \mathbf{m}: \mathscr{D} \rightarrow \mathbb{R}^{d}$ and $\mathbf{b}: \mathscr{D} \rightarrow \mathbb{R}^{d}$ such that $z:=(u, \mathbf{m}, \mathbf{b})$ satisfies the constraint

$$
z(y) \in K_{y}:= \begin{cases}\left\{(u, \mathbf{m}, \mathbf{b})|\mathbf{m}=u \mathbf{b}, \quad| \mathbf{b} \mid=1, \quad u^{2}=F(y)\right\} & \text { if } y \in \mathscr{U} \\ 0 & \text { if } y \in \mathscr{D} \backslash \mathscr{U}\end{cases}
$$

for a.e. $y=(x, t) \in \mathscr{D}$.

Suppose that $z=(u, \mathbf{m}, \mathbf{b}) \in L^{\infty}(\mathscr{D})$ satisfies (14) and (15) in sense of distributions and moreover (16) holds a.e. in $\mathscr{D}$. Then the couple $(u, \mathbf{b})$ satisfies the assertion of Theorem 2.

Let us check the assumption of Theorem 2 .

Lemma 5. Suppose that $A, B \subset \mathbb{R}^{n}$ are compact sets and $r>0$ is such that

- for any $z \in A$ there exists $z^{\prime} \in B \cap B_{r}(z)$ 
- for any $z \in B$ there exists $z^{\prime} \in A \cap B_{r}(z)$

Then $d_{\mathcal{H}}(A, B)<r$.

Proof. Suppose that $d_{\mathcal{H}}(A, B) \geq r$. Then without loss of generality we can assume that there exists $z \in A$ such that for any $z^{\prime} \in B$ we have $z \notin B_{r}\left(z^{\prime}\right)$. But then the ball $B_{r}(z)$ cannot contain any point of $B$, which leads to a contradiction.

Lemma 6. If $F: \mathscr{U} \rightarrow \mathbb{R}$ is continuous, bounded and non-negative then the map $y \mapsto K_{y}$ is continuous and bounded (w.r.t. $\left.d_{\mathcal{H}}\right)$ on $\mathscr{U}$.

Proof. Let $f(y):=\sqrt{F(y)}$. Let us fix $y \in \mathscr{U}$. For any $\varepsilon>0$ let $\delta>0$ be such that $\left|f(y)-f\left(y^{\prime}\right)\right|<\varepsilon$ for any $y^{\prime} \in B_{\delta}(y) \subset \mathscr{U}$. Let us prove that $d_{\mathcal{H}}\left(K_{y}, K_{y^{\prime}}\right)<2 \varepsilon$ for all $y^{\prime} \in B_{\delta}(y)$.

For any $z \in K_{y}$ there exist $\sigma \in\{ \pm 1\}$ and $\mathbf{b} \in \mathbb{R}^{d}$ with $|\mathbf{b}|=1$ such that $z=(\sigma f(y), \sigma f(y) \mathbf{b}, \mathbf{b})$. Then $z^{\prime}:=\left(\sigma f\left(y^{\prime}\right), \sigma f\left(y^{\prime}\right) \mathbf{b}, \mathbf{b}\right)$ belongs to $K_{y^{\prime}}$ and $\left|z-z^{\prime}\right| \leq 2\left|f(y)-f\left(y^{\prime}\right)\right|$. Hence there exists $z^{\prime} \in K_{y^{\prime}} \cap B_{2 \varepsilon}(z)$.

Similarly, for any $z^{\prime} \in K_{y^{\prime}}$ there exist $\sigma \in\{ \pm 1\}$ and $\mathbf{b} \in \mathbb{R}^{d}$ with $|\mathbf{b}|=1$ such that $z^{\prime}=$ $\left(\sigma f\left(y^{\prime}\right), \sigma f\left(y^{\prime}\right) \mathbf{b}, \mathbf{b}\right)$. Then $z:=(\sigma f(y), \sigma f(y) \mathbf{b}, \mathbf{b})$ belongs to $K_{y}$ and $\left|z-z^{\prime}\right| \leq 2\left|f(y)-f\left(y^{\prime}\right)\right|$. Hence there exists $z \in K_{y} \cap B_{2 \varepsilon}\left(z^{\prime}\right)$.

Therefore by Lemma 5 we have $d_{\mathcal{H}}\left(K_{y}, K_{y^{\prime}}\right)<2 \varepsilon$.

Lemma 7. Assumption 1 holds for the system (14)-(16).

Proof. Let $\phi: \mathscr{D} \rightarrow \mathbb{R}$ be a non-negative smooth function such that $0 \leq \phi \leq 1$ on $\mathscr{D}, \phi=0$ on $\mathscr{D} \backslash B_{1}(0)$ and $\phi=1$ on $B_{1 / 2}(0)$.

Part 1. Suppose that $d>2$. Let us show that Assumption 1 holds with $\Lambda=\mathbb{R}^{2 d+1}$. Fix $\bar{u} \in \mathbb{R}$, $\overline{\mathbf{m}} \in \mathbb{R}^{d}$ and $\overline{\mathbf{b}} \in \mathbb{R}^{d}$ and let $\bar{z}=(\bar{u}, \overline{\mathbf{m}}, \overline{\mathbf{b}})$. Since $d>2$ there exists a unit vector $\mathbf{n} \in \mathbb{R}^{d}$ such that $\mathbf{n} \cdot \overline{\mathbf{m}}=\mathbf{n} \cdot \overline{\mathbf{b}}=0$. Denote $\hat{\mathbf{n}}=(0, \mathbf{n}), \overline{\mathbf{a}}=(\bar{u}, \overline{\mathbf{m}})$. For any $k \in \mathbb{N}$ define $\overline{\mathbf{a}}_{k}: \mathscr{D} \rightarrow \mathbb{R}^{d+1}$ by

$$
\overline{\mathbf{a}}_{k}(y):=\overline{\mathbf{a}}\left(\hat{\mathbf{n}} \cdot \nabla_{y}\left(\phi \Pi_{k}\right)\right)-\hat{\mathbf{n}}\left(\overline{\mathbf{a}} \cdot \nabla_{y}\left(\phi \Pi_{k}\right)\right)
$$

where $y=(t, x)$ and

Observe that

$$
\Pi_{k}(y):=\frac{\sin (k \hat{\mathbf{n}} \cdot y)}{k} .
$$

$$
\operatorname{div}_{y} \overline{\mathbf{a}}_{k}=\left(\overline{\mathbf{a}} \cdot \nabla_{y}\right)\left(\hat{\mathbf{n}} \cdot \nabla_{y}\right)\left(\phi \Pi_{k}\right)-\left(\hat{\mathbf{n}} \cdot \nabla_{y}\right)\left(\overline{\mathbf{a}} \cdot \nabla_{y}\right)\left(\phi \Pi_{k}\right)=0 .
$$

Let $\left(u_{k}, \mathbf{m}_{k}\right)$ denote the components of $\overline{\mathbf{a}}_{k}$, then by the equation above we have $\partial_{t} u_{k}+\operatorname{div}_{x} \mathbf{m}_{k}=0$. Similarly let

$$
\mathbf{b}_{k}(t, x):=\overline{\mathbf{b}}\left(\mathbf{n} \cdot \nabla_{x}\left(\phi \Pi_{k}\right)\right)-\mathbf{n}\left(\overline{\mathbf{b}} \cdot \nabla_{x}\left(\phi \Pi_{k}\right)\right)
$$

Then arguing as above $\operatorname{div} \mathbf{b}_{k}=0$.

Now we introduce $w_{k}:=\left(u_{k}, \mathbf{m}_{k}, \mathbf{b}_{k}\right)$. Then

$$
w_{k}(y)=\bar{z} \phi \cos (k \hat{\mathbf{n}} \cdot y)+f \Pi_{k}
$$

where $f$ does not depend on $k$ and vanishes on $B_{1 / 2}(0)$.

On the other hand,

$$
\begin{aligned}
\int_{\mathscr{D}}\left|w_{k}\right|^{2} d y \geq \int_{B_{1 / 2}(0)}\left|w_{k}\right|^{2} d y=\int_{B_{1 / 2}(0)}|\bar{z}|^{2} \cos ^{2}(k \hat{\mathbf{n}} \cdot y) d y= \\
=\int_{B_{1 / 2}(0)}|\bar{z}|^{2} \frac{1+\cos (2 k \hat{\mathbf{n}} \cdot y)}{2} d y \geq \frac{|\bar{z}|^{2}}{4}\left|B_{1 / 2}(0)\right|
\end{aligned}
$$

provided that $k$ is sufficiently large. 
Part 2. Suppose that $d=2$ and fix $\bar{z}=(\bar{u}, \overline{\mathbf{m}}, \overline{\mathbf{b}}) \in \mathbb{R} \times \mathbb{R}^{2} \times \mathbb{R}^{2}$ with $\bar{u} \neq 0$. Let us look for a localized plane wave in the following form:

$$
w_{k}=\left(\mathbf{a}_{k}, \mathbf{b}_{k}\right)
$$

with

$$
\begin{aligned}
& \mathbf{a}_{k}(y)=\nabla_{y} \times\left(\phi \mathbf{A} \frac{\sin (k \mathbf{n} \cdot y)}{k}\right) \\
& \mathbf{b}_{k}(t, x)=\nabla_{x}^{\perp}\left(\phi \frac{\sin (k \mathbf{n} \cdot(t, x))}{k}\right)
\end{aligned}
$$

where $\mathbf{n}=\left(n_{t}, \mathbf{n}_{x}\right) \in \mathbb{R} \times \mathbb{R}^{2}$ and $\mathbf{A} \in \mathbb{R}^{3}$ are to be chosen and $k \in \mathbb{N}$. Then, by construction

$$
\operatorname{div}_{y} \mathbf{a}_{k}=0, \quad \operatorname{div}_{x} \mathbf{b}_{k}=0 .
$$

Then, we get

$$
w_{k}=\hat{z} \phi \cos (k \mathbf{n} \cdot y)+f \frac{\sin (k \mathbf{n} \cdot y)}{k}
$$

where $\hat{z}=\left(\mathbf{A} \times \mathbf{n}, \mathbf{n}_{x}^{\perp}\right)$ and $f$ does not depend on $k$ and vanishes on $B_{1 / 2}(0)$.

In order to have $\hat{z}=\bar{z}$ the vectors $\mathbf{A}$ and $\mathbf{n}$ must satisfy

$$
\begin{aligned}
\mathbf{A} \times \mathbf{n} & =(\bar{u}, \overline{\mathbf{m}}), \\
\mathbf{n}_{x}^{\perp} & =\overline{\mathbf{b}} .
\end{aligned}
$$

From the second equation we immediately obtain that $\mathbf{n}_{x}=-\overline{\mathbf{b}}^{\perp}$. Since $\bar{u} \neq 0$ there exists $n_{t}$ such that $\mathbf{n} \perp(\bar{u}, \overline{\mathbf{m}})$. Then, we can always find $\mathbf{A}$ such that the first equation is satisfied. It remains to observe that the estimate (17) holds also in the considered case. We thus have verified Assumption 1 for $\Lambda=\mathbb{R}^{5} \backslash\{\bar{u}=0\}$.

Proof of Theorem 2. By symmetry of $K_{y}$ for any $y \in \mathscr{U}$ we have $0 \in \operatorname{int}$ conv $K_{y}$. On the other hand $K_{y}=\{0\}$ for any $y \in \mathscr{D} \backslash \mathscr{U}$. Therefore $u \equiv 0, \mathbf{m} \equiv 0$ and $\mathbf{b} \equiv 0$ is a subsolution of (14)-(16). Then the result follows from Lemma 2, Lemma 7 and Theorem 1 .

\section{REFERENCES}

[ABC13] G. Alberti, S. Bianchini, and G. Crippa, Structure of level sets and Sard-type properties of Lipschitz maps: results and counterexamples, Ann. Scuola Norm. Sup. Pisa Cl. Sci. 5 (2013), 863-902.

[ABC14] _ A uniqueness result for the continuity equation in two dimensions, J. Eur. Math. Soc. (JEMS) 16 (2014), 201-234.

[AC14] L. Ambrosio and G. Crippa, Continuity equations and ODE flows with non-smooth velocity, Proc. Roy. Soc. Edinburgh Sect. A 144 (2014), no. 6, 1191-1244.

[ADLM07] L. Ambrosio, C. De Lellis, and J. Malý, On the chain rule for the divergence of BV-like vector fields: applications, partial results, open problems, Perspectives in nonlinear partial differential equations, Contemp. Math., vol. 446, Amer. Math. Soc., Providence, RI, 2007, pp. 31-67.

[Aiz78] M. Aizenman, On vector fields as generators of flows: A counterexample to Nelson's conjecture, Ann. Math. 107 (1978), 287-296.

[Amb] L. Ambrosio, Transport equation and Cauchy problem for BV vector fields, Invent. Math.

[BG14] S. Bianchini and N. A. Gusev, Steady nearly incompressible vector fields in 2d: chain rule and renormalization, Preprint (2014).

[BLFNL] A. Bronzi, M. Lopes Filho, and H. Nussenzveig Lopes, Wild solutions for 2d incompressible ideal flow with passive tracer, Preprint.

[Bog07] V. I. Bogachev, Measure theory. Vol. I, II, Springer-Verlag, Berlin, 2007.

[CFG11] D. Cordoba, D. Faraco, and F. Gancedo, Lack of uniqueness for weak solutions of the incompressible porous media equation, Arch. Ration. Mech. Anal. 200 (2011), no. 3, 725-746.

[CGSW] G. Crippa, N. A. Gusev, S. Spirito, and E. Wiedemann, Failure of the chain rule for the divergence of bounded vector fields, Preprint. 
[CLR03] F. Colombini, T. Luo, and J. Rauch, Uniqueness and nonuniqueness for nonsmooth divergence free transport, Seminaire: Équations aux Dérivées Partielles, 2002-2003, Sémin. Équ. Dériv. Partielles, Exp. No. XXII, École Polytech., Palaiseau, 2003, pp. 1-21.

[Dep03] N. Depauw, Non unicité des solutions bornées pour un champ de vecteurs BV en dehors d'un hyperplan, C. R. Math. Acad. Sci. Paris 337 (2003), no. 4, 249-252.

[DL89] R. J. DiPerna and P.-L. Lions, Ordinary differential equations, transport theory and Sobolev spaces, Invent. Math. 98 (1989), no. 3, 511-547.

[DL08] C. De Lellis, Ordinary differential equations with rough coefficients and the renormalization theorem of Ambrosio [after Ambrosio, DiPerna, Lions], Astérisque (2008), no. 317, Exp. No. 972, viii, 175-203, Séminaire Bourbaki. Vol. 2006/2007.

[DLS09] C. De Lellis and L. Székelyhidi, Jr., The Euler equations as a differential inclusion, Ann. of Math. 170 (2009), no. 3, 1417-1436.

[DLS10] _ On admissibility criteria for weak solutions of the Euler equations, Arch. Ration. Mech. Anal. 195 (2010), no. 1, 225-260.

[DLS12] _ The h-principle and the equations of fluid dynamics, Bull. Amer. Math. Soc. (N.S.) 49 (2012), no. $3,347-375$.

[Gus11] N. A. Gusev, Continuity equation as a differential inclusion, Unpublished (2011).

[Oxt80] J. Oxtoby, Measure and category, second ed., Graduate Texts in Mathematics, vol. 2, Springer-Verlag, New York-Berlin, 1980, A survey of the analogies between topological and measure spaces.

[Sch93] V. Scheffer, An inviscid flow with compact support in space-time, J. Geom. Anal. 3 (1993), no. 4, 343-401.

[Shn97] A. Shnirelman, On the nonuniqueness of weak solution of the Euler equation, Comm. Pure Appl. Math. 50 (1997), no. 12, 1261-1286.

[Shv11] R. Shvydkoy, Convex integration for a class of active scalar equations, J. Amer. Math. Soc. 24 (2011), no. 4, 1159-1174. MR 2813340 (2012d:35295)

[Szé12] L. Székelyhidi, Jr., Relaxation of the incompressible porous media equation, Ann. Sci. Éc. Norm. Supér. (4) 45 (2012), no. 3, 491-509.

(G. Crippa) Departement Mathematik und Informatik, Universität Basel, Rheinsprung 21, CH-4051 BASEL, SWITZERLAND

E-mail address: gianluca.crippa@unibas.ch

(N. Gusev) Dybenko st., 22/3, 94, 125475 Moscow, Russia

E-mail address: n.a.gusev@gmail.com

(S. Spirito) GSSi - Gran Sasso Science Institute, Viale Francesco Crispi 7, 67100 L'Aquila, Italy E-mail address: stefano.spirito@gssi.infn.it

(E. Wiedemann) Hausdorff Center for Mathematics and Mathematical Institute, Universität Bonn, Endenicher Allee 60, 53115 Bonn, Germany

E-mail address: emil.wiedemann@hcm.uni-bonn.de 